\title{
Análisis multidimensional de la Inteligencia Emocional
}

\section{Multidimensional Analysis of Emotional Intelligence}

DOI: $10.46932 / s f j d v 2 n 5-109$

Received in: Oct 1st, 2021

Accepted in: Dec 30th, 2021

\author{
Roberto García Sánchez \\ Doctor en Historia de la Psiquiatría \\ Profesor de Psicología de la Personalidad \\ Universidad de La Laguna \\ Campus de Guajara, s/n, 38071 San Cristóbal de La Laguna, Santa Cruz de Tenerife \\ E-mail: extrgarcias@ull.edu.es \\ Laura Romero de los Reyes \\ Graduada en Psicología \\ Universidad de La Laguna \\ Campus de Guajara, s/n, 38071 San Cristóbal de La Laguna, Santa Cruz de Tenerife \\ E-mail: laurardlr@hotmail.com
}

\section{RESUMEN}

Existen diferentes modelos teóricos de Inteligencia Emocional (IE), que explica por qué y cómo argumentar sobre la autenticidad de la IE. Destacan algunos teóricos, como Mayer y Salovey, que creen que los conceptos científicos son concretos y restrictivos, y una vez que dejen de serlo, se convertirán en revelaciones acientíficas. Los modelos de habilidades enfatizan la importancia del procesamiento cognitivo de la IE, mientras que otros abogan por un modelo de IE amplio que integre todos los aspectos de la inteligencia académica que no se han considerado, como el control de impulsos o la automotivación. Además, hay autores que consideran que la inteligencia emocional es un rasgo de personalidad necesario para adaptarse al entorno y para una vida exitosa. Los expertos en la materia utilizan habilidades, rasgos o métodos híbridos, que se consideran enfoques diferentes del mismo tema. Esto los llevó a adoptar una estructura de IE diferente, porque el uso de diferentes modelos generó conflictos en los resultados de la investigación científica. Los avances de la neurociencia han permitido verificar la existencia de la IE, entendida como un conjunto de habilidades distintas de las cognitivas.

Después de realizar esta revisión, la conclusión es que existen diferencias en el concepto de IE del autor.

Palabras clave: inteligencia emocional, modelos teóricos, personalidad, rasgo, habilidades.

\begin{abstract}
There are different theoretical models of Emotional Intelligence (EI), which explains why and how to argue about the authenticity of EI. Some theorists stand out, such as Mayer and Salovey, who believe that scientific concepts are concrete and restrictive, and once they cease to be, they will become unscientific revelations. Skills models emphasize the importance of EI cognitive processing, while others advocate a broad EI model that integrates all aspects of academic intelligence that have not been considered, such as impulse control or self-motivation.

In addition, there are authors who consider emotional intelligence is a personality trait necessary to adapt to the environment and for a successful life. Hybrid skills, traits, or methods are used by experts in the field, which are considered different approaches to the same topic. This led them to adopt a different EI
\end{abstract}


structure, because the use of different models generated conflicts in the results of scientific research. Advances in neuroscience have made it possible to verify the existence of EI, understood as a set of skills other than cognitive.

After conducting this review, the conclusion is that there are differences in the author's concept of EI.

Keywords: emotional intelligence, theoretical models, personality, trait, abilities.

\section{INTRODUCCIÓN}

La sociedad ha tenido muy en cuenta en los últimos años un ideal específico del ser humano: ser inteligente. En la escuela tradicional se consideraba inteligente a un niño cuando dominaba las lenguas clásicas, latín o griego, matemáticas, etc. Posteriormente, se ha considerado inteligente al niño que obtiene una puntuación alta en las pruebas de inteligencia.

El cociente intelectual (CI) se ha convertido en el referente de este ideal y todo ello se mantiene por la relación entre el CI y el rendimiento académico: los estudiantes que obtienen las puntuaciones más altas en las pruebas de CI tienden a obtener las mejores notas en la escuela, como regla general.

Hoy en día, este punto de vista ha sido cuestionado por dos razones. En primer lugar, la inteligencia académica no es suficiente para lograr el éxito laboral. Los abogados que ganen más casos, los médicos más prestigiosos, los profesores más brillantes, los emprendedores más exitosos, los gerentes que logren mejores resultados no necesariamente tienen que ser los más inteligentes de su promoción, desde el punto de vista cognitivo. No tienen por qué ser esos adolescentes que siempre levantaron el mano primero en clase cuando les preguntó su maestro o que destacaron por sus altas calificaciones académicas en la escuela secundaria y bachillerato. No tenían por qué ser esos adolescentes que estaban solos en el recreo mientras otros jugaban o hablaban. Sin embargo, hay altas posibilidades de que fueran quienes supieron reconocer sus emociones y cómo manejarlas adecuadamente para apoyar su inteligencia; fueron los que sembraron las relaciones sociales y los que aprendieron a dominar los mecanismos que motivan y orientan a las personas; fueron los que se interesaron más por las personas que por las cosas y entendieron la importancia de lo que se llama capital humano.

En segundo lugar, la inteligencia no garantiza el éxito en la vida diaria. La inteligencia no garantiza la felicidad en nuestras relaciones amorosas, familiares o de amistad. El cociente intelectual de las personas no contribuye al equilibrio emocional ni a la salud mental. Son otras habilidades emocionales y sociales las que permiten la estabilidad emocional y mental, así como la adaptación social.

Este es un contexto adecuado para que la sociedad se pregunte, ¿por qué son necesarias las emociones en la vida? La respuesta, obviamente, no es simple, pero ha permitido el desarrollo de modelos alternativos. Por tanto, se ha sustituido el ideal tradicional de persona inteligente, dando lugar al concepto de inteligencia emocional (IE) como forma alternativa. En la literatura científica hay dos modelos 
principales de IE: los modelos mixtos y el modelo de capacidad. Los modelos mixtos combinan dimensiones de personalidad como el optimismo y la automotivación con habilidades emocionales (Goleman, 1998; Bar-On, 1997).

En nuestro país, España, el que más difusión ha tenido en contextos educativos ha sido el modelo de inteligencia emocional de Daniel Goleman, relacionado más con razones de marketing que con la literatura científica. John Mayer y Peter Salovey, desarrollaron el modelo de habilidad, menos conocido en nuestro medio, pero con gran respaldo empírico en revistas especializadas. De hecho, Goleman tomó el concepto de IE de un artículo de Mayer y Salovey del año 1990, aunque en su libro le da un punto de vista diferente.

El modelo de habilidades de Mayer y Salovey se centra en el procesamiento emocional de la información y el estudio de las habilidades relacionadas con dicho procesamiento. A partir de esta teoría, la IE se define como la capacidad de las personas para atender y percibir los sentimientos de forma adecuada y precisa, la capacidad de asimilarlos y comprenderlos adecuadamente y la capacidad de regular y modificar nuestro estado de ánimo o el de los demás. el resto.

A partir de este modelo, la IE involucra cuatro componentes principales:

- Percepción y expresión emocional: reconocer conscientemente las emociones e identificar cómo se siente y poder darle una etiqueta verbal.

- Facilitación emocional: capacidad de generar sentimientos que faciliten el pensamiento.

- Comprensión emocional: integrar lo sentido en el pensamiento y saber considerar la complejidad de los cambios emocionales.

- Regulación emocional: dirigir y gestionar las emociones, tanto positivas como negativas, de forma eficaz.

Estas capacidades están íntima y necesariamente interrelacionadas, a tal punto que para una adecuada regulación emocional es necesaria una buena comprensión emocional y, al mismo tiempo, para una adecuada comprensión se requiere una adecuada percepción de las emociones. Por tanto, el proceso contrario no siempre es cierto, ya que hay personas con una gran capacidad de percepción emocional que en ocasiones carecen de comprensión y regulación emocional.

Esta habilidad se puede utilizar en uno mismo (competencia personal o inteligencia intrapersonal) o en otros (competencia social o inteligencia interpersonal). En este sentido, la IE se diferencia de la inteligencia social y las habilidades sociales en que incorpora emociones internas privadas que son importantes para el desarrollo personal y el ajuste emocional. Por otro lado, las áreas personal e interpersonal son bastante independientes y no siempre se desarrollan por concatenación. Hay personas con una gran capacidad de comprensión y regulación de sus emociones y muy equilibradas emocionalmente, pero con pocas habilidades para conectar con los demás. También ocurre lo contrario, 
hay personas con una enorme capacidad empática para comprender a los demás, pero que no son muy hábiles para controlar y regular sus propias emociones.

La inteligencia emocional, según el modelo de capacidad, no puede entenderse ni como un rasgo de personalidad ni como parte del carácter de una persona, por ejemplo, si un individuo tiene la extraversión como rasgo de personalidad, ¿podría el grado de inteligencia emocional personal o interpersonal que tiene? La respuesta es no. Otro tema es la posibilidad de que exista una interrelación entre la IE y la personalidad, tal como existe con la inteligencia abstracta.

Teniendo en cuenta lo anterior, las personas con cierto tipo de personalidad desarrollarán más o menos fácilmente, más o menos rápidamente, sus habilidades emocionales. Al fin y al cabo, la persona no es la suma de sus partes, sino una fusión que convive, milagrosamente, de forma integrada.

\section{INTELIGENCIA EMOCIONAL EN LA ESCUELA}

No cabe duda de que la importancia de las emociones en la vida de las personas es suficiente para que las escuelas y toda la comunidad educativa reciban educación en inteligencia emocional. Sin embargo, hay muchas otras razones por las que las personas deberían educar sus emociones desde la escuela, como dijo Darder (2003):

\footnotetext{
"Es importante mejorar la comprensión del mundo emocional y las relaciones interpersonales, y pasar de una intervención intuitiva a una intervención más planeado para lograr las capacidades adecuadas de inteligencia emocional. La falta de control emocional afecta el desempeño escolar, genera inseguridades ante situaciones, actitud de rechazo por parte de los compañeros, falta de concentración en una tarea..."
}

Conocer las emociones significa que debe hacerse en el momento en que ocurren, esto es fundamental para que el alumno que se encuentra en las escuelas desarrolle correctamente las habilidades emocionales, ya que puede concienciar a las personas de la situación en ese momento, permitir que los alumnos la conozcan y transmitir ciertas emociones. La reacción que tiene un alumno ante situaciones muestra cómo se enfrenta a la realidad y su entorno. En otras palabras, la educación emocional puede ayudar a los estudiantes a descubrir el estado actual, conectarlo con otras personas en su vida diaria y ayudarlos a darse cuenta de que las reacciones positivas a las emociones pueden aumentar los estados felices y prepararlos para emociones futuras.

Además, como dice Gómez-Bruguera (2003), la inteligencia emocional da a los niños un sentimiento necesario para hacerlos sentir compromiso interno, seguridad, autoconfianza y libertad por el trabajo que están realizando en ese momento. Quienes desarrollan una buena inteligencia emocional terminan estableciendo una buena relación con otras personas, por otro lado, también les permite ser más animados, creativos y tranquilos. 
La educación emocional debe comenzar lo antes posible, Gómez-Bruguera (2003) señaló que la situación ideal es estudiar la inteligencia emocional de 0 a 3 años, porque durante este período, las emociones son más naturales, sinceras y espontáneas, y no ser manipulado por experiencia, no por vergüenza, no por timidez. En definitiva, consistiría en enseñar a los niños el equilibrio emocional y la felicidad, sabiendo que la edad de 2 a 4 años es fundamental para la tarea de socializar al niño.

Codina (2003) señaló en su artículo que desconocer los aspectos emocionales de los niños en el aprendizaje escolar en todos los sectores es grave, pero esto es especialmente cierto en los grupos más vulnerables, porque significa que es imposible alcanzar el nivel general. desarrollo que el niño o cualquier alumno de todas las etapas de educación y desarrollo necesita, pues si un niño no recibe el tratamiento emocional adecuado a nivel social y emocional, se sentirá inseguro, lo que se traducirá en miedo y ansiedad.

Por otro lado, para la estructura mental de los niños con problemas emocionales, el pensamiento abstracto es mínimo y la estructura de la personalidad también se ve afectada, pues los niños con baja inteligencia emocional tienden a permanecer en el presente sin experimentar el pasado y el futuro, lo que afectará la memoria y el futuro. la capacidad de resistir situaciones graves...

Finalmente, Goleman (2017) afirmó que la inteligencia emocional es decisiva y, en ocasiones, incluso más importante que el cociente intelectual (CI). Saber que una persona ha logrado buenos resultados solo nos brindará información sobre su desempeño sobresaliente en las pruebas de conocimiento, pero no nos dará información sobre cómo se desempeñará ante las dificultades, pues el CI no brinda ninguna preparación para la resistencia a situaciones graves, ni cómo afrontarlas.

La inteligencia emocional en la escuela es una dificultad que hay que afrontar a lo largo de la vida. Más importante aún, dijo Goleman (2007), existe una clara evidencia de que las personas desarrolladas emocionalmente tienen ventajas sobre otras en todos los aspectos de la vida, porque tienden a sentirse más satisfechas, efectivas y capaces, en última instancia, más productivas. Por otro lado, aquellos que no pueden controlar sus emociones se involucrarán constantemente en luchas internas, reduciendo su capacidad y productividad. Además, hacer trabajo emocional significa cultivar cinco habilidades básicas para el desarrollo integral de una persona, que es el principal objetivo de la escuela:

- Conocer las propias emociones: la capacidad de reconocer los sentimientos cuando aparecen.

- La capacidad de controlar las emociones: darse cuenta de que puede controlar los sentimientos y adaptarse al momento en que suceden, para dar una correcta salida a las emociones.

- Habilidades de automotivación: controlar las emociones es fundamental para mantener la concentración, la motivación y la creatividad.

- Conocer las emociones de otras personas: la empatía y la comprensión son habilidades básicas. 
- Control de relaciones: la capacidad de conectarse con los demás y sus emociones de manera adecuada.

Los estudiantes pasan la mayor parte de su infancia y adolescencia en el aula. Este período es fundamentalmente importante para el desarrollo emocional, por lo que el entorno escolar se configura como un espacio privilegiado para la interacción emocional y social. El docente se convierte en su referente en cuanto a actitud, comportamiento, emoción y sentimiento, más importante, por eso la profesión de educadores y formadores es una de las profesiones más importantes, pero en muchos casos, el riesgo de hacerlo mal es producir el efecto contrario al esperado.

Los maestros que saben cómo adaptarse y reunirse con los estudiantes pueden ayudar a reducir la ira, el miedo y la frustración durante el día escolar; el ambiente es generalmente menos agresivo, lo que hace que el trato sea más humano. Por el contrario, los profesores que a menudo desprecian a los estudiantes no deben sorprenderse de que los estudiantes pisoteen inconscientemente las emociones de sus compañeros desfavorecidos, ya que los niños aprenden a expresar emociones al observar el desempeño de sus adultos más cercanos (padres y educadores), lo que se conoce como aprendizaje vicario.

Por tanto, las habilidades cognitivas y emocionales de los docentes afectarán el crecimiento intelectual y emocional de los estudiantes, por ejemplo, cuando un docente tiene una baja percepción de un estudiante, este último lo sentirá y se encontrará en una clara desventaja frente a las opiniones de profesores, expertos y profesores oficialmente reconocidos. Con el tiempo, el alumno finalmente acepta las opiniones negativas del profesor y actúa como un mal alumno.

Si se piensa, por un lado, que el docente no ve resultados positivos en los alumnos, entonces este problema no se puede cambiar y, por otro lado, si el docente no señala sus limitaciones y no les ayuda, entonces los estudiantes no progresarán con los que contar. Este tipo de problema educativo se está extendiendo: los docentes que no comprenden el concepto de estudiante eventualmente los despreciarán y alienarán, y es posible que a su vez intenten cambiar el plan de estudios o el nivel, pero con el tiempo los docentes se sentirán insatisfechos con los estudiantes. estudiantes. Finalmente, los juicios de valor despectivos sobre los estudiantes se han convertido en autoevaluaciones negativas por parte de los profesores. Por lo tanto, en un sentido amplio, los docentes pueden y deben ser siempre educadores fundamentales, más que jueces o miembros de tribunales de calificación, sus acciones no deben limitarse a sanciones por mala conducta o evaluación indiferente de los conocimientos de los estudiantes. El diálogo entre profesores y alumnos es fundamental.

Entender al alumno como una persona con una dimensión mayor que la de un simple alumno es fundamental para comprender sus problemas y poder ayudarlos a resolverlos. Los docentes también son responsables del nivel de autoestima académica de los estudiantes a través de la docencia, evaluación y valoración de su desempeño. Además, si en determinadas circunstancias se hace una explicación negativa 
de sus intenciones y habilidades, puede incluso llevarlos a despreciarse a sí mismos. Finalmente, desde un punto de vista emocional, los estudiantes con maestros talentosos prefieren ir a la escuela, no tienen miedo de aprender y desarrollar una autoestima saludable, pero lo más importante, la humanidad de los maestros hace que los estudiantes y los padres se destaquen. Porque los maestros son modelos a seguir tanto para los estudiantes como para sus padres. Siempre que sean personas conocedoras de las emociones, siendo la forma ideal de observar, razonar y reaccionar ante la vida, serán modelos a seguir para los estudiantes.

En primer lugar, el profesor debe asumir el papel de padre del alumno, que será un modelo insustituible de inteligencia emocional. Junto a la enseñanza de conocimientos teóricos y valores, los docentes tienen otro aspecto igualmente importante, que es moldear y ajustar las emociones características que se dan en el aula.

\section{INTELIGENCIA EMOCIONAL EN EL CONTEXTO FAMILIAR}

Las emociones forman parte de la vida de una persona, la forma en la que comprenden, reconocen y expresan sus propias emociones y comprenden las emociones de los demás, estará ligada a la educación emocional inmersa en el entorno familiar, esto es lo que llamamos inteligencia emocional familiar. Dentro de la familia se sientan las bases para el desarrollo emocional y social de los niños, por lo que su influencia en este sentido es crucial. La familia es un excelente entorno social, donde se crean los primeros lazos emocionales y alrededor de ellos se construye el modelo de apego que determina nuestras relaciones futuras. Las personas más cercanas al niño serán modelos a seguir, transmitiendo sin saberlo la forma de expresar la emoción, la forma de hablar de la emoción, la forma de pensar la emoción y la forma de actuar sobre la emoción.

Si hay un entorno donde se deben desarrollar las habilidades emocionales, es el familiar. El fuerte vínculo emocional entre padres e hijos significa que ambos deben aprender a ser emocionalmente inteligentes para crear una vida mejor para todos. Diferentes estudios han demostrado que las relaciones interpersonales (incluidas las familiares) son uno de los predictores del bienestar emocional y el bienestar en general. Curiosamente, estas relaciones son también la principal causa de conflicto e incomodidad, que pueden provocar emociones negativas como tristeza, resentimiento, odio, etc. La clave está en ser emocionalmente inteligente y desarrollar y utilizar estas habilidades a la hora de interactuar. Los padres, al ser modelos a seguir para los niños, el primer paso consistiría en la conciencia emocional de los padres, es decir, comprender sus propias emociones, causas y posibles consecuencias. El siguiente paso es ayudar a los niños a descubrir cómo se sienten, cuanto antes comience este viaje con ellos, mejor. Es importante enseñar a los niños a conectarse con ellos mismos para que puedan comprender mejor sus sentimientos, y cualquier momento o situación del día ayuda a practicar y desarrollar la conciencia emocional. Altamente 
relevante es prestar atención a sus emociones, ya sean positivas (por ejemplo, alegría) o negativas (por ejemplo, tristeza o enojo) para que puedan ser etiquetadas y nombradas más adelante. A continuación, es necesario trabajar con ellos para producir emociones, y de esta manera tener conocimiento sobre qué los hizo sentir así. Cabe destacar que todas las emociones son necesarias, deben ser aceptadas, por lo tanto, donde se debe actuar es el comportamiento que de ellas deriva.

La educación en inteligencia emocional significará prestar atención a las emociones de los niños y responder a sus necesidades emocionales, lo que traerá importantes beneficios para su crecimiento, pero también traerá importantes consecuencias positivas para los adultos, el clima y las funciones familiares.

Las familias con un alto nivel de inteligencia emocional tienen algunas características en común:

- Los familiares saben cómo expresar sus emociones.

- Pueden comprender las emociones de los demás.

- Tienen un entorno ideal para la comunicación y la confianza.

- Se apoyan mutuamente.

- Nadie se siente juzgado, criticado o etiquetado.

- Emiten amor y felicidad.

Durante décadas, el mundo de las emociones y los sentimientos se ha atribuido al contexto, porque la gente cree que las emociones son mucho menos importantes que el conocimiento racional, pero hoy vivimos en una época en la que el ámbito emocional parece estar enfatizando su importancia respecto a cualquier otra habilidad humana.

Pero, por ejemplo, la educación emocional no solo debe llevarse a cabo en el ámbito escolar, sino que como el ser humano siempre es emocional, hay muchos entornos implicados (familia, compañeros, diversión, etc.). La familia, como primer lugar social para interactuar con los niños, también debe saber cómo enseñar una buena educación emocional. En el futuro, cuando los niños interactúen con sus compañeros, les permitirá llevar a cabo el tipo de aprendizaje emocional que se creó en casa y responderán emocionalmente al comportamiento de ellos, lo que a su vez brindará retroalimentación emocional a los miembros de la familia (Extremera, 2003). El propósito de la educación es desarrollar plenamente la personalidad general del individuo, para ello debe desarrollarse cognitiva y emocionalmente. Las personas con una buena comprensión y manejo de las emociones tienen más probabilidades de sentirse satisfechas y más efectivas en todos los aspectos de la vida (Martín, Blanco \& Rubio, 2011). En este sentido, la familia tiene una gran relación, porque en la familia, cuando se encuentra por primera vez enfado, enfado, tensión y otras situaciones, es decir, cuando el niño desobedece, las emociones negativas son más difíciles de controlar. Los padres deben predicar con el ejemplo regulando sus emociones, en lugar de dejándose llevar por los primeros impulsos; el diálogo y las respuestas alternativas son una buena solución a los primeros pensamientos que pueden ser más impulsivos. 
Los padres pueden decir que juegan un papel vital como primer elemento social en la educación emocional de los niños, porque este es el punto de partida para establecer la relación inicial y vínculo emocional con el niño, pues este imitará o generalizará las acciones que ve de sus padres más de lo que ellos creen, por lo tanto, es importante que los padres estén formados emocionalmente. Los progenitores, como ya se mencionó, deben recibir una buena educación emocional, porque si saben reconocer bien sus emociones, facilitará que los niños comprendan lo que está sucediendo y, al ser conscientes de sus emociones, pueden ayudar a los niños a mejorar su inteligencia emocional al identificar, nombrar, comprender y regularlas.

Como ejemplo, podemos hablar del enfado de los niños cuando no consiguen lo que quieren (Ibarrola, 2003), que suele ser lo más común en la educación infantil. Cuando no le dice al niño si quiere algo o no compra algo, el niño se enojará, lo que a menudo se manifiesta en forma de rabietas. Lo importante es que los padres deben entender a sus hijos y saber cómo responderán cuando les prohíban algo, no les dejen hacer algo o les nieguen algo.

En otras situaciones, los niños pueden sentir miedo y mostrar miedo en su comportamiento, por ejemplo, cuando fallan en una actividad o no hacen algo, lo que los lleva a la frustración y la ira (Ibarrola, 2003). Para ellos, lo importante es estar seguros y volver a proponer actividades similares, lo que les dará miedo de hacerlo y si no se puede hacer les causará enfado, pero aprenderán a desarrollar tolerancia a la frustración y resiliencia. Es de suma importancia enseñar a los bebés a utilizar técnicas de relajación, por ejemplo, para que estén más tranquilos, para poder empezar de nuevo, para estar más relajados, sin ningún miedo, sin provocar estrés por rabia o frustración. Se le puede recomendar que se detenga por un momento, respire y vuelva a intentarlo.

Los celos son otra situación en la que los niños están enojados. La celotipia es una emoción compleja caracterizada por el miedo a perder o ver disminuido el afecto de los seres queridos por la presencia de otros (Ibarrola, 2003). Como no hay tiempo suficiente para cuidar a los niños, la aparición de un nuevo hermano puede despertar la ira de los niños, porque hay un nuevo integrante que también necesita cuidados. Por ello, es importante que los padres mantengan una conversación con sus hijos mayores y les informen sobre la futura llegada de sus hermanos y les enseñen a no enojarse, porque una buena historia implicará un abordaje diferente de la situación (Ibarrola, 2003). Tomando como ejemplo la relación familiar entre padres e hijos, es necesario darse cuenta de la importancia de formar a los padres en temas emocionales y también en los aspectos más importantes de la paternidad. 


\section{RELACIÓN ENTRE LOS CONTEXTOS SOCIOEDUCATIVOS Y SU INFLUENCIA EN LA INTELIGENCIA EMOCIONAL}

En la actualidad, en esta sociedad y en la escuela, la emoción no ha recibido la atención que merece, se han tratado y trabajado más los contenidos académicos y se han dejado de lado todos los aspectos de la educación emocional. Investigarlos es importante porque el conocimiento académico no es suficiente para lograr el éxito profesional.

Según Qualter, Whiteley, Hutchinson y Pope (2007) lo citado por estos autores, se ha demostrado que un buen desarrollo y educación emocional están relacionados con un desarrollo académico adecuado, es decir, estos niños tendrán más éxito en el futuro.

Por tanto, a las emociones se les debe dar el valor agregado que tienen en la formación integral de los niños y, para lograr este objetivo, se debe promover la educación emocional, que se entiende como un proceso educativo continuo dirigido a promover la emoción y, además, su desarrollo. La competencia es un elemento básico del desarrollo humano, con el propósito de mantenerlo vivo y mejorar el bienestar personal y social (Bisquerra, 2000). Como afirma Bisquerra, la educación emocional debe promoverse a lo largo de la vida, ya que permitirá el desarrollo del carácter personal en general y también ayudará a dominar las habilidades emocionales para adaptarse a diversas situaciones.

Según la investigación de Bisquerra (2003), los objetivos de la educación emocional son los siguientes:

- Conocer mejor las propias emociones.

- Reconocer las emociones ajenas.

- Desarrollar la capacidad de regular las emociones.

- Prevenir los efectos nocivos de las emociones negativas.

- Cultivar la capacidad de generar emociones positivas.

- Desarrollar habilidades de automotivación.

- Adoptar una actitud positiva ante la vida.

Sin embargo, como ya se ha visto, para llevar a cabo una buena educación emocional uno de los objetivos es desarrollar la capacidad de regular las propias emociones, ya que esto puede evitar muchos problemas que surgen al interactuar con los demás. La capacidad para regular las emociones consiste en modular o controlar la propia respuesta ante situaciones intensas, sean positivas o negativas (FernandezBerrocal \& Extremera, 2000).

Los niños que sepan autorregularse emocionalmente sabrán afrontar nuevas situaciones en lugar de confundirse con el primer impulso, y serán capaces de generar y mostrar reacciones alternativas para controlar sus emociones. Además, regular las emociones hará sentir bien de manera saludable, lo cual es sumamente importante porque estas afectan a la salud mental. Según la definición de Punset (2008), si no 
entendemos cómo nos sentimos y por qué nos sentimos así, no seremos capaces de entender por qué pensamos y actuamos de una determinada manera. Pero para convertirte en una persona con buena inteligencia emocional y capacidad para regular las emociones, primero debes saber qué emociones se están sintiendo, es decir, qué va a pasar y, para ello, es necesario identificar cuál es la sensación de que estás teniendo.

Como afirma Cristina Muñoz (2007), “cuanto más nos acercamos a definir un sentimiento (por ejemplo: alegría), mejor conocimiento tenemos y, por tanto, más sana es la forma en que se relaciona y puede ser manejado por uno mismo. y con los demás". La educación en inteligencia emocional debe convertirse en uno de los principales objetivos del campo educativo. Se recomienda llevar a cabo una formación clara y curricular en este tipo de capacidades a través de asignaturas que incluyan y enfaticen habilidades para mejorar las habilidades emocionales.

En esta dirección, el docente no solo debe enfocarse en enseñar modelos de comportamiento correcto, sino también prestar atención a las emociones, consiste en comprender el comportamiento del alumno desde una perspectiva emocional más que conductual y también se trata de enseñar una forma de trabajar los sentidos en una forma inteligente de entrenar emociones. En el contexto de la educación, los profesores se convierten en los principales líderes de las emociones de los estudiantes, lo que significa dar ejemplo. Los docentes que sean capaces de detectar, comprender y regular las emociones del grupo de clase lograrán su equilibrio emocional. Se necesita capacitación de maestros para completar un plan de estudios que carece de habilidades de desarrollo para manejar pensamientos, emociones y sentimientos, y la adquisición de habilidades para comprender mejor la propia realidad y mejorar las relaciones personales y profesionales, en última instancia, lo que hace que las vidas sean más seguras y felices.

Según la definición de Salovey y Mayer en 1990, la inteligencia emocional es "una forma de inteligencia social que significa ser capaz de orientar los sentimientos y emociones de uno mismo y de los demás, saber distinguir entre ellos y utilizar esta información y propio comportamiento".

En los últimos años, la investigación sobre la influencia de la IE en el equilibrio psicológico y la felicidad personal de los estudiantes ha despertado un gran interés. La mayoría de estos estudios hacen referencia a las recomendaciones de Salovey y Mayer (1997), que definen la IE según cuatro componentes (Fernández-Berrocal et al., 2002):

- Percepción: una buena percepción significa saber leer los propios sentimientos y emociones, identificarlos y experimentarlos. Al identificar los sentimientos a través de buenas órdenes, se sientan las bases para aprender a controlarse, regular las propias reacciones y no dejarse llevar por un gran impulso o entusiasmo.

- Asimilación: las emociones y los pensamientos se fusionan, si se sabe cómo usar las emociones para ayudar a los pensamientos, esto ayudará a razonar con más sabiduría y a tomar mejores 
decisiones. Capturar emociones y mediarlas con pensamientos ayuda a adaptarse al entorno de manera más adecuada.

- Comprensión: para comprender los sentimientos de los demás, se debe empezar por aprender a comprenderse a uno mismo. Si se reconocen los propios sentimientos, se tendrá mayor facilidad para conectar emocionalmente con los demás.

- Regulación: una de las habilidades más difíciles de mostrar y dominar es la de regular el estado emocional. Incluye la capacidad de regular o controlar una respuesta emocional en situaciones intensas (positivas o negativas). Se cree que la regulación de las emociones es la capacidad de evitar respuestas emocionales incontroladas en situaciones de provocación o miedo, aunque el campo se está expandiendo hacia la autorregulación de las emociones positivas.

Los componentes de la IE incluyen la capacidad de percibir, comprender y gestionar de forma adaptativa las propias emociones. La falta de habilidades de inteligencia emocional afecta a los estudiantes tanto dentro como fuera del entorno educativo, y contribuye a cuatro aspectos básicos de los problemas de conducta en los estudiantes (Fernández-Berrocal et al., 2008).

- IE y relaciones interpersonales: una IE más alta puede ayudar a proporcionar suficiente información sobre el estado mental de quienes nos rodean. Para controlar el estado emocional de los demás, primero se debe poder manejar bien los propios estados emocionales. Las personas emocionalmente inteligentes no solo son más capaces de percibir, comprender y manejar sus propias emociones, sino que también pueden inferir las emociones de los demás. Por ello, la IE juega un papel fundamental en la generación, mantenimiento y calidad de las relaciones interpersonales.

- IE y Salud Mental: los estudios han proporcionado un marco adecuado para comprender los procesos emocionales básicos que constituyen un adecuado equilibrio psicológico y ayudar a comprender mejor el papel mediador de ciertas variables emocionales en los estudiantes, su ajuste psicológico y el bienestar que afectan a las personas, como en los modelos Mayer y Salovey.

- IE y rendimiento académico: poder enfocarse en las emociones que siente la persona, no huir de los sentimientos que está teniendo y poder eliminar las emociones negativas tendrá un papel decisivo en la salud mental de los estudiantes. El equilibrio psicológico está relacionado y afectará al rendimiento académico final. Las personas con capacidades de regulación emocional más débiles tienen más probabilidades de tener estrés y problemas emocionales en el proceso de aprendizaje, por lo que se verán favorecidas por el uso de habilidades adaptativas que les permitan hacer frente a tales dificultades.

- La aparición de la IE y la conducta destructiva: las habilidades contenidas en la IE son un factor clave para prevenir la aparición de conductas destructivas que derivan en problemas emocionales. 
Los estudiantes con niveles más bajos de IE muestran un mayor nivel de impulsividad y menores habilidades interpersonales y sociales, lo que conduce a la posible generación de diversas conductas antisociales.

A los estudiantes que tienen la capacidad de regular las emociones negativas y mantener las positivas les resultará más fácil desarrollar habilidades más detalladas relacionadas con la tolerancia a la frustración y la confianza en sí mismos, como aceptar las críticas, defender la propia posición de forma no agresiva, etc. (Fernández-Berrocal \& Extremera, 2002).

\section{CONCLUSIÓN}

El propósito de la educación es formar alumnos emocionalmente competentes (capaces de reconocer y gestionar sus emociones) y, por tanto, de relacionarse con los demás de forma adecuada y pacífica. Es aquí donde nace el enfoque de la educación emocional como una forma de involucrar al proceso educativo en la consecución de este objetivo.

La educación emocional se refiere al desarrollo de las competencias emocionales a través de una programación sistemática y progresiva, de acuerdo con las edades de los estudiantes y que incluye y acompaña el aprendizaje de conocimientos y habilidades. En las escuelas, este enfoque es necesario desde el nivel más bajo hasta el último de los estudiantes, es decir, en todos los niveles educativos y en todas las etapas de desarrollo. Debe ser un aprendizaje centrado en el desarrollo afectivo y mediado por la educación, no como una actividad extraescolar, sino como algo universal en la práctica docente, pues no solo los tutores se encargan de trabajar los temas afectivos, todos los profesores interactúan también con los estudiantes. El docente emocionalmente inteligente es, por tanto, el encargado de formar y educar al alumno en habilidades como el conocimiento de las propias emociones, el desarrollo del autocontrol y la capacidad de expresar adecuadamente sus sentimientos a los demás. Para que el docente esté preparado para asumir este desafío, es necesario que piense en su propio desarrollo emocional, solo así podrá formarse y adquirir herramientas metodológicas que le permitan realizar este trabajo.

Un factor que debemos tener en cuenta es el de educar emocional y moralmente a los alumnos si no tienen una estructura de valores establecida, además de un determinado dominio de las propias emociones. Un docente emocionalmente inteligente debe contar con suficientes recursos emocionales que acompañen el desarrollo afectivo de sus alumnos, ya que con eso establece un vínculo sano y estrecho con ellos, comprende sus estados emocionales y les enseña a conocerse y a resolver los conflictos cotidianos de forma serena y tranquila.

Vivas de Chacón (2004) realizó una investigación sobre las competencias socioemocionales del docente, con el fin de proponer un programa de formación. A partir de los datos recolectados en un 
conjunto de entrevistas realizadas con los ellos, propone un modelo que identifica cuatro tipos de necesidades que los docentes consideran como competencias que aún deben desarrollarse:

- Conocimientos sobre inteligencia emocional: sobre las emociones y su relación con los procesos cognitivos, así como el papel que desempeña la IE en la adaptación y establecimiento interpersonal de las personas.

- Habilidades interpersonales: para identificar las emociones de sus alumnos, percibir sus estados de ánimo, escuchar, ser empático, tomar decisiones, resolver conflictos, tener liderazgo y capacidad para dirigir y persuadir, descubrir las fortalezas de los demás, ser justos y equitativos.

- Habilidades intrapersonales: para controlar, gestionar e interpretar las propias emociones, para que se pueda reaccionar de forma coherente con ellas.

- Habilidades didácticas para la educación emocional: nuevas habilidades profesionales para un modelado más efectivo, para estimular el desarrollo armonioso de los estudiantes y abordar los problemas emocionales; fomentar el desarrollo de competencias didácticas creativas que promuevan escuelas emocionalmente inteligentes que construyan entornos propicios y estimulantes para el desarrollo afectivo.

Un docente emocionalmente inteligente debe ser capaz de interpretar estados afectivos para orientarlos de una manera útil en el aprendizaje, enfocándose en sus habilidades interpersonales y de liderazgo. Un maestro motivador, conciliador y con buen sentido del humor tendrá un impacto positivo en sus alumnos. Por el contrario, un profesor poco tolerante, rígido y con poco uso de las emociones puede entorpecer el clima del aula. 


\section{REFERENCIAS}

Bar-On, R. (1997). The Emotional Quotient inventory (EQ-I): Techical Manual. Toronto. Canada: Multi-Health Systems.

Bisquerra R. (2003). Emotional education and basic skills for life. Research Journal, 21 (1), 7-43.

Bisquerra, R. (2000). Emotional education and well-being. Barcelona: Praxis.

Codina, A. (2003). Introduction to emotional intelligence for managerial work. In virtual library of the Latin American Foundation for Quality. [Consulted on May 20, 2006 at: http://www.calidad.org/public/ arti2002 / 1034460306_alexis.htm].

Darder, P. (2003). Emotions and education: what they are and how to intervene from school. Madrid: Graó.

Fernández-Berrocal P. \& Extremera N. (2002) Emotional intelligence as an essential skill in school. OEI-IberoAmerican Journal of Education, 29 (1), 1-6.

Fernández-Berrocal, P. \& Extremera, N. (2000). Emotional intelligence as an essential skill in school. IberoAmerican Journal of Education, 29, 1- 6

Fernández-Berrocal, P. \& Extremera, N. (2008). Emotional intelligence and health. In Mestre, J.M. and FernándezBerrocal, P. (Eds), Manual de Inteligencia emotional, (pp. 173-187). Madrid: Ed. Pyramid.

Goleman, D. (1998). Working with emotional intelligence, New York: Bantman.

Goleman, D. (2007). Emotional intelligence. Barcelona: Kairos.

Gómez-Bruguera, J. (2003). Emotional education and language at school. Barcelona: Octaedro.

Ibarrola B. (2003) Stories to feel. Educate emotions, Madrid: Ediciones SM.

Martin Vicente R., Blanco Rodríguez H. \& Rubio Hernández M.L (2011). Project and emotional education. Website: http://www.caib.es/sacmicrofront/archivopub.do?ctrl=MCRST151ZI113641\&id=113641.

Mayer, J. D. \& Salovey, P. (1997). What is emotional intelligence? In P. Salovey and D. Sluyter (Eds), Emotional Development and Emotional Intelligence: Implications for Educators (pp. 3-31). New York: Basic Books.

Muñoz, C. (2007) Emotional intelligence: the secret for a happy family. Edited by: General Family Directorate. Comunity of Madrid.

Punset E. (2008). Compass for emotional boaters. Spain. Bulk Editorial.

Qualter, P., Whiteley, H. E., Hutchinson, J. M., \& Pope, D. J. (2007). Supporting the development of emotional intelligence competencies to ease the transition from primary to high school. Educational Psychology in Practice, 23 (1), 79-95.

Salovey, P. \& Mayer, J.D. (1990). Emotional Intelligence. Imagination, Cognition, and Personality, 9: 185-211.

Vivas de Chacón, M. (2004). The socio-emotional competences of the teacher: a look from the trainers of trainers. I University Conference Socio-professional Competences of Education Degrees. Venezuela. 